\title{
In vivo Assessment of the Hepatotoxicity of Mixed Gemcitabine and 5-Fluorouracil Loaded on Microemulsion in Mice Bearing Ehrlich Ascites Carcinoma
}

\author{
Mayson H. Alkhatib ${ }^{1, *}$, May M. Alasmari ${ }^{1}$, Faiza Abdo ${ }^{2}$ \\ ${ }^{1}$ Department of Biochemistry, King Abdulaziz University, Jeddah, Saudi Arabia \\ ${ }^{2}$ Department of Biological Sciences, King Abdulaziz University, Jeddah, Saudi Arabia
}

Copyright $\bigcirc 2017$ by authors, all rights reserved. Authors agree that this article remains permanently open access under the terms of the Creative Commons Attribution License 4.0 International License

\begin{abstract}
A combination of two or more chemotherapeutic agents in a nanoparticle is a promising approach to improve the treatment effectiveness and reduce their toxicity. In this study, the formulations of $30 \mathrm{mg} / \mathrm{kg}$ of gemcitabine (GEM) mixed with $2.5 \mathrm{mg} / \mathrm{kg}$ of 5 -fluorouracil (5-FU), either loaded on a microemulsion (GEM+5'FU-ME) or dissolved in water (GEM+5' $\mathrm{FU}-\mathrm{Sol})$, were examined to explore their antitumor activity in mice inoculated with Ehrlich ascites carcinoma (EAC). Eighty mice were split into eight groups (10 mice/group). Group I served as the untreated EAC bearing mice. Groups II-VIII were EAC bearing mice treated with free GEM (GEM-Sol), GEM-loaded-ME (GEM-ME), free 5-FU (5'FU-Sol), 5-FU-loaded-ME (5'FU-ME), GEM+5'FU-Sol, GEM+5'FU-ME and the blank-ME (B-ME), respectively. The hepatotoxicity was identified by determining the serum enzymes, and studying the histological changes of the liver tissues. The z-average diameter and zeta potential of the nanodroplets of GEM+5'FU-ME were $122.1 \pm 26.80 \mathrm{~nm}$ and $-4.40 \pm 1.76 \mathrm{mV}$, respectively. Results demonstrated that drug-loaded ME formulations have lowered the enzyme levels of ALT, AST and ALP and did not cause damage in the hepatocytes when compared to the drug solution formulations. In conclusion, the combination treatments of GEM and 5-FU in a ME may protect the drugs from degradation in the liver.
\end{abstract}

Keywords Chemotherapeutic Agents, Histology, Z-Average Diameter, Zeta Potential, Antitumor Activity

\section{Introduction}

Chemotherapeutic agents are antitumor drugs used to treat cancer by mainly inducing apoptosis in the cells. Due to the lack of specificity of the anticancer agents to the cancer cells, their mechanism of action is usually associated with severe side effects. As a consequence, many drugs with antitumor activities are toxic to the liver at which most of them get metabolized [1]. Gemcitabine (GEM) is a chemotherapeutic agent used to treat various types of cancer [2]. Due to its rapid clearance in the urine, GEM is used in high doses in order to improve its therapeutic index. Additionally, GEM encounters resistance from many cancers because of the loss of transporters and kinases. Therefore, GEM administration is associated with adverse side effects that would affect the liver. 5-FU is a halogenated pyrimidine in which fluorine at position 5 allows the molecule to mimic uracil biochemically, which enables it to act as antimetabolite. It is the thymidylate synthase enzyme inhibitor, which gets converted into 5-fluorodeoxyuridylates, which disrupts RNA synthesis in addition to inhibiting the enzyme [3]. Previous researches have proposed to combine both of GEM and 5-FU since they have different mechanism of actions and thereby mixing them could lead to a synergistic effect that hold greater efficacy $[4,5]$. However, the main issue with the combination of the chemotherapeutic agents is their effect on the hepatocytes of the liver [1].

Microemulsion (ME), a colloidal system that consists of water, oil, surfactant and/or cosurfactant, has many advantages including its simplicity and spontaneity of preparation, transparency, ability to solubilize a wide range of drugs, and improve drug bioavailability and permeation through the biological membranes. These advantages make ME remarkable drug delivery systems [6]. The present study was conducted to evaluate the hepatotoxicity of the combination of GEM and 5-FU loaded on a ME delivery system in Ehrlich ascites carcinoma (EAC) bearing female 
Swiss albino mice. The prepared ME formulations were physically characterized by the Zetasizer. The toxicities of the ME formulations were identified by measuring the serum biochemical parameters and studying the histological changes.

\section{Materials and Methods}

\subsection{Materials}

Polyoxyethylene sorbitan monooleate (tween80), Isopropyl Myristat (IPM), Ethanol, Sorbitan monolaurate (Span20), 5-FU and GEM hydrochloride were purchased from Sigma (Missouri, US). Serum kits were obtained from Human Biochemical and Diagnostic (Wiesbaden, Germany).

\subsection{Subjects}

80 healthy female Swiss albino mice (25-35g) were acclimatized in accordance with King Abdulaziz University's policy and the international ethical guidelines on the care and use of laboratory animals [7]. The ethical approval was obtained from the research ethics committee in the Faculty of Medicine at King Abdulaziz University. EAC cells were procured from American Type Tissue Culture Collection (Manassas, VA, USA).

\subsection{Preparation of the ME Formulations}

The blank ME formulation (B-ME) was prepared as mentioned by [8]. Briefly, B-ME formulation was formed by mixing $50 \%$ of the oil phase, IPM, $10 \%$ of the aqueous phase, consisted of $60 \%$ of distilled water and $40 \%$ of ethanol, and $40 \%$ of surfactant mixtures of tween 80 and span 20 at a ratio of 3:2, respectively. GEM-loaded-ME (GEM-ME) was prepared by dissolving a $30 \mathrm{mg}$ of $\mathrm{GEM} / \mathrm{kg}$ of mouse in $0.2 \mathrm{ml}$ of ME. The 5 -FU formulas were produced by dissolving $5 \mathrm{mg}$ of $5-\mathrm{FU} / \mathrm{kg}$ of mouse in $0.2 \mathrm{ml}$ of ME (5'FU-ME) or water ( $\left.5^{\prime} \mathrm{FU}-\mathrm{Sol}\right)$. The combination of GEM and 5-FU formulas were prepared by mixing $30 \mathrm{mg}$ GEM $/ \mathrm{kg}$ of mouse and $2.5 \mathrm{mg}$ of $5-\mathrm{FU} / \mathrm{kg}$ of mouse in $0.2 \mathrm{ml}$ of $\mathrm{ME}\left(\mathrm{GEM}+5^{\prime} \mathrm{FU}-\mathrm{ME}\right)$ or water (GEM+5'FU-Sol). The formula of GEM dissolved in distilled water (GEM-Sol) was prepared by dissolving $60 \mathrm{mg}$ of GEM/ $\mathrm{kg}$ of mouse in $0.2 \mathrm{ml}$ of distilled water.

\subsection{Characterization of the ME Formulations}

The z-average diameter, polydispersity index (PDI), zeta potential of the ME formulations (B-ME, GEM-ME, GEM+5'FU-ME and 5'FU-ME) were measured by using Zetasizer (3,000 HS; Malvern Instruments, Malvern, UK). The desired ME formula was prepared by diluting a $100 \mu \mathrm{l}$ of the sample in a $400 \mu 1$ of Tris buffer ( $\mathrm{pH} 7.2)$.

\subsection{Inoculation of EAC in the Mice}

The mice were split into eight groups $(\mathrm{n}=20)$ according to the administered drug, as shown in Table 1. The body weight of all mice was taken before injection with EAC cells maintained in the ascetic form as described by [9] All of the mice in each group were injected intraperitoneally with $2.5 \times 10^{6} \mathrm{EAC}$ cells/mouse on the 0 th day. Group I served as the control. After $72 \mathrm{~h}$ of tumor inoculation, animals in Groups II-VIII were treated with different routes and doses of the desired drug, given every three days.

Table 1. The examined mice groups $(n=20)$ according to the administered drug, route and dose

\begin{tabular}{|c|c|c|c|}
\hline Group number & Drug administrated & Route & Dose/72h \\
\hline I & EAC control -Untreated & - & - \\
\hline II & GEM-ME & Oral & 2 \\
\hline III & GEM-Sol & Injection & 4 \\
\hline IV & 5'FU-ME & Oral & 4 \\
\hline V & 5'FU-Sol & Injection & 4 \\
\hline VI & GEM+5'FU-ME & Oral & 2 \\
\hline VII & GEM+5'FU-Sol & Injection & 3 \\
\hline VIII & B-ME & Oral & 4 \\
\hline
\end{tabular}

\subsubsection{Serum Analysis}

On the 17th day, the body weight of each mouse was recorded after 12 hours fasting. The blood, collected by the retro orbital plexus method, was utilized for analyzing the serum by measuring the alkaline phosphatase (ALP), aspartate aminotransferase (AST), alanine aminotransferase (ALT), albumin (ALB), total protein (TP), total and direct bilirubin (BIL).

\subsubsection{Histology}

Immediately following sacrificing the mice on the 17th day after the blood collection, the collected liver tissues were incubated in a fixation buffered formalin and were treated as described elsewhere [9].

\subsection{Statistical Analysis}

All values were expressed as mean $\pm \mathrm{SD}\left(\mathrm{X}^{-} \pm \mathrm{SD}\right)$ obtained from the experiments $(\mathrm{n}=10)$. Statistical analysis was performed with one-way analysis of variance (ANOVA) test and independent sample t-test using the MegaStat Excel (version 10.3, Butler University).

\section{Results}

\subsection{Characterization of the ME Formulations}

The physical characteristics of the ME formulations in terms of the z-average diameter, zeta potential and 
polydispersity index were displayed in Figure 1. The z-average diameters of the B-ME $(99.70 \pm 0.762 \mathrm{~nm})$ was comparable to the diameter of GEM-ME (109.30 \pm $2.22 \mathrm{~nm})$. In contrast, the z-average diameter of B-ME has greatly enlarged when loaded with 5-FU (5'FU-ME) to be $164.00 \pm 3.49 \mathrm{~nm}$ but it has moderately increased when the ME was loaded with the combination of 5- FU and GEM $\left(\mathrm{GEM}+5^{\prime} \mathrm{FU}-\mathrm{ME}\right)$ to be $122.10 \pm 3.00 \mathrm{~nm}$. The zeta potentials of the formulations were comparable and have negative charges, excluding GEM+5'FU-ME that showed a low value of zeta potential. In contrast, the polydispersity indices of all of the drug-loaded-MEs formulations have significantly increased compared to B-ME.
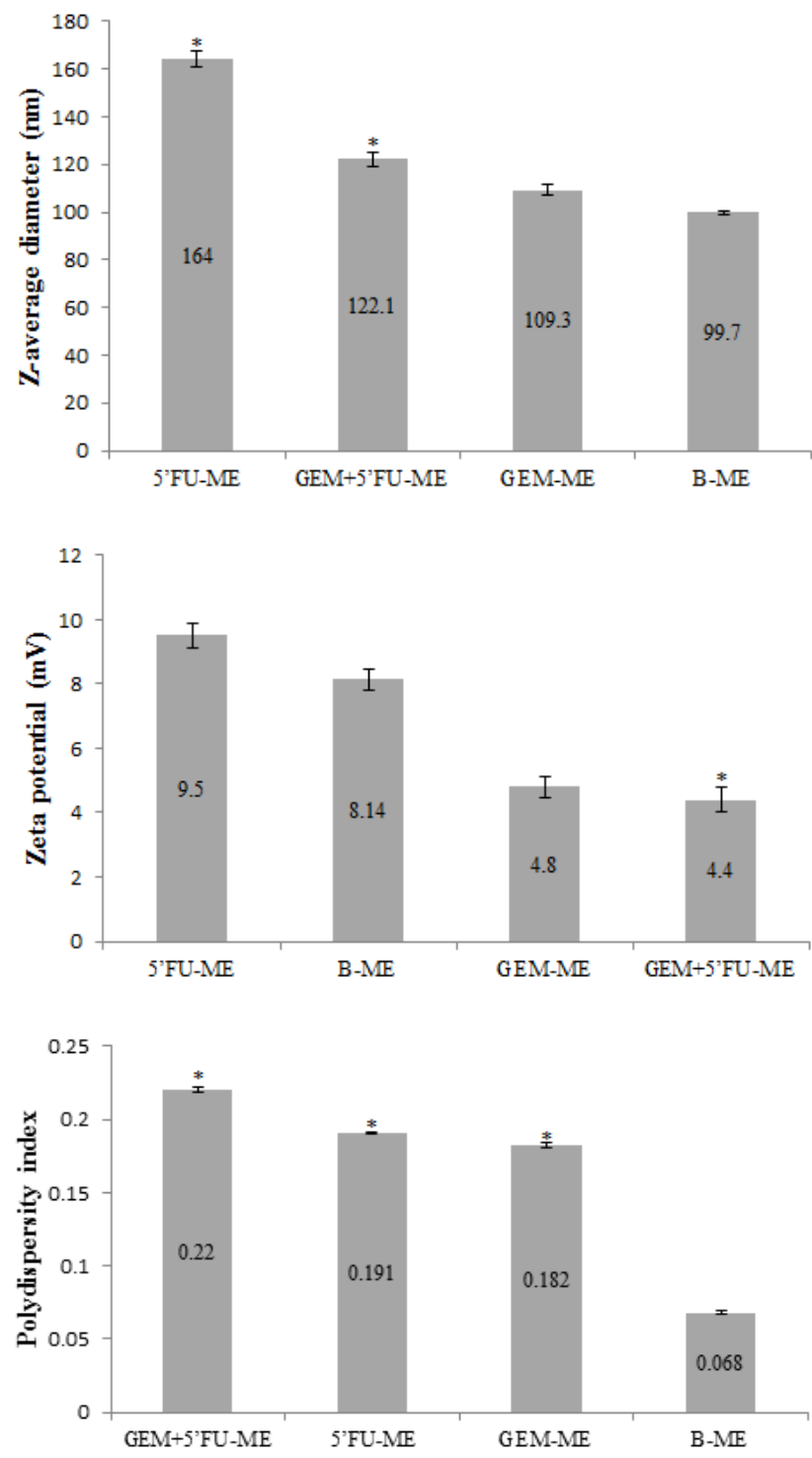

Figure 1. The physical characteristics of the nanodroplets of the ME formulations, it should note that the zeta potentials were negatively charged. Error bars represent the standard error of the mean $(n=20)$. The significant differences were classified according to P-value as (1) significant $(*)$; and (2) very highly significant $(* * *)$.

\subsection{Hepatotoxicity of the ME Formulations}

\subsubsection{Serum Analysis}

Figure 2 displays the measured levels of the ALP, ALT and AST of the tested animals. It has been found that the levels of ALP, ALT and AST were lowered when the drug was loaded in the ME relative to the solution formulation. It should be noted that B-ME group has comparable levels of ALP, ALT and AST levels in the EAC group.
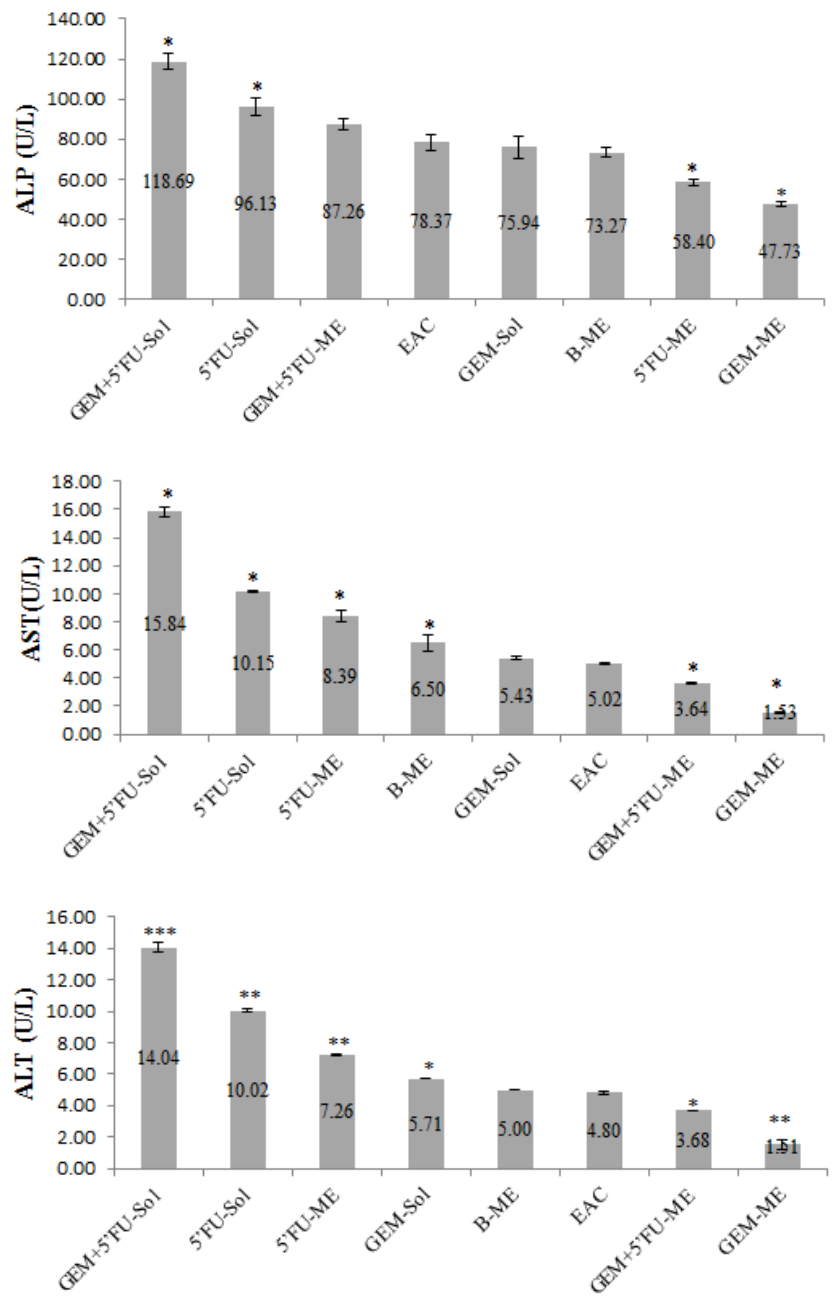

Figure 2. The levels of ALP, ALT and AST of the tested animal groups, Error bars represent the standard error of the mean $(n=5)$. The significant differences were classified according to P-value as (1) significant $(*)$; and (2) highly significant $(* *)$.

Furthermore, Table 2 illustrates the amounts of total protein (TP), albumin (ALB), direct bilirubin (D.BIL) and total bilirubin (T.BIL) of the tested animals. Compared to the EAC group, the TP amount was only raised in the GEM-Sol group and has got considerably reduced in GEM+5'FU-Sol group. In contrast, the amounts of ALB, D.BIL and T.BIL did not differ in all of the tested groups.

\subsubsection{Liver Histology}

Figure 3A, presented the normal hepatic structure of the liver section of the normal group examined under light 
microscope where the hepatocytes appear arranged in single-cell thick plates separated by vascular sinusoids with no vacuoles closely linked with a venous portal system (central venous) with normal presences of Kupffer cells. In contrast, the hepatic tissue of the untreated EAC bearing mice, showed wider vascular sinusoids and the appearance of more Kupffer cells as shown in Figure 3B. The mice treated with B-ME showed a hepatic tissue similar to normal group, the hepatocytes in normal shape, having a nucleus in their center, with the presence of few Kupffer cells, narrow vascular sinusoids and a central vein as shown in Figure 4 The hepatic tissue of the mice treated with GEM-ME, shown in Figure 5A, exhibited central veins filled with blood, normal hepatocytes with nucleus at their center, normal structure sinusoidal spaces, activation of Kupffer cells and stimulation of vacuolization, whereas mice treated with GEM-Sol revealed tissues with damaged hepatic cells that lack nuclei inside it as displayed in Figure 5B. As well, for the 5'FU-ME and 5'FU-Sol, shown in Figure 6, the hepatic tissue appeared in normal form around central vein, a dilated sinusoidal space, and normal activation of Kupffer cells for the mice treated with 5'FU-ME while the tissues of 5'FU-Sol group have hepatocytes that endured death due to the shrinkage of the nuclei. Regarding the tissues of the combination formulas groups, the tissue of the GEM+5'FU-ME group, viewed in Figure 7A, showed a normal hepatocytes, the excessive formation of the Kupffer cells, dilated sinusoids and hemorrhage in central vein. In contrast, the liver tissue of the GEM+5'FU-Sol group, shown in Figure 7B, exhibited an enormous number of damaged hepatocytes that lost their regular shapes and their nuclei besides the presence of normal hepatocytes, moderate formation of Kupffer cells and more dilated blood sinusoids spaces.

Table 2. The serum analysis of the EAC bearing mice treated with different drug formulations in order to detect the liver function

\begin{tabular}{|c|c|c|c|c|}
\hline Group & TP $(6.6-8.7 \mathrm{~g} / \mathrm{dL})$ & ALB $(3.8-5.1 \mathrm{~g} / \mathrm{dL})$ & D.BIL $(0.25 \mathrm{mg} / \mathrm{dL})$ & T.BIL $(1.1 \mathrm{mg} / \mathrm{dL})$ \\
\hline EAC & $6.789 \pm 1.025$ & $4.120 \pm 1.02$ & $0.172 \pm 0.021$ & $0.814 \pm 0.15$ \\
\hline GEM-Sol & $8.459 \pm 0.925^{*}$ & $4.159 \pm 0.96$ & $0.267 \pm 0.032$ & $1.330 \pm 0.167$ \\
\hline GEM-ME & $6.655 \pm 0.75$ & $3.139 \pm 1.20$ & $0.146 \pm 0.044$ & $0.716 \pm 0.23$ \\
\hline 5'FU-Sol & $7.491 \pm 0.47$ & $3.883 \pm 0.25$ & $0.287 \pm 0.052$ & $1.017 \pm 0.14$ \\
\hline 5'FU-ME & $6.088 \pm 1.25$ & $3.811 \pm 0.50$ & $0.262 \pm 0.152$ & $1.197 \pm 0.28$ \\
\hline GEM+5'FU-Sol & $3.749 \pm 0.525^{* * *}$ & $4.310 \pm 0.27$ & $0.226 \pm 0.065$ & $1.270 \pm 0.45$ \\
\hline GEM+5'FU-ME & $7.322 \pm 0.825$ & $3.883 \pm 0.35$ & $0.196 \pm 0.057$ & $1.189 \pm 0.64$ \\
\hline B-ME & $5.014 \pm 1.25$ & $3.788 \pm 0.50$ & $0.136 \pm 0.101$ & $1.042 \pm 0.81$ \\
\hline
\end{tabular}

Data were expressed as mean \pm Standard deviation. (1) If $0.01 \leqslant$ P-value $<0.05$, the difference between samples is significant $(*)$; (2) If the P-value $<0.001$, the difference between samples is very highly significant $(* * *)$.
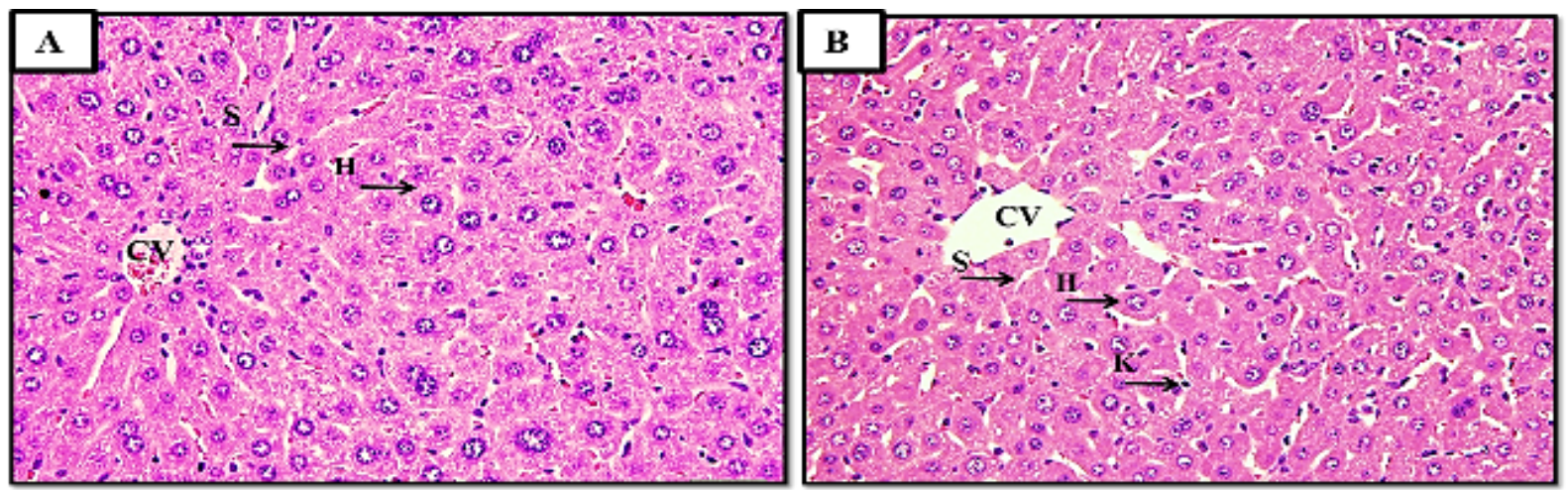

Figure 3. Photomicrograph of the hepatic tissue of the A) normal group, showing the hepatocytes $(\mathrm{H})$ that are arranged in cords radiating from the central vein (CV) and separated by blood sinusoids (S), and B) EAC group, showing the central vein (CV) with hepatocytes (H), excessive dilated blood sinusoids (S), and the presence of few Kupffer cells (K). H\&E x 400 


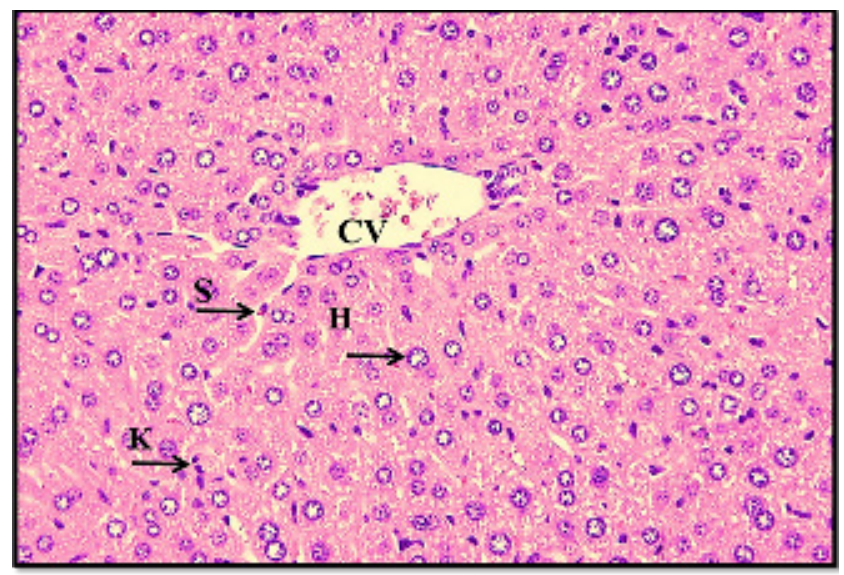

Figure 4. Photomicrograph of the hepatic tissue of the B-ME group, showing a central vein with hemorrhage (CV), hepatocytes $(\mathrm{H})$ rounded to polyhedral in shape, having nucleus in their center, less Kupffer cells (K) and narrow sinusoids (S). H\&E x 400
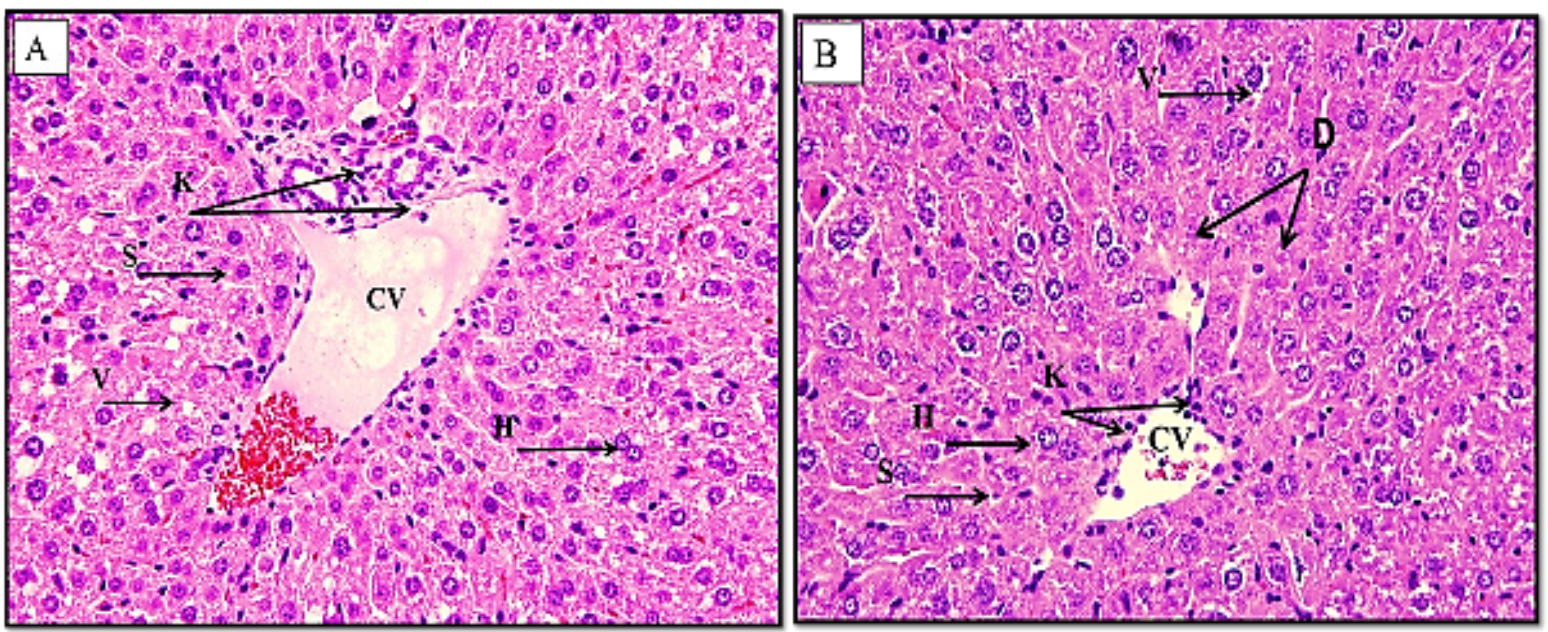

Figure 5. Photomicrograph of hepatic tissue of A) GEM-ME group, showing hepatocytes (H) rounded in shape with vacuoles (V), central vein (CV) filled with blood, activation of Kupffer cells (K) near to normal structure sinusoidal spaces (S), and B) GEM-Sol group, showing damaged hepatocytes (D) that lack nuclei, hepatocytes $(\mathrm{H})$ containing vacuoles $(\mathrm{V})$, dilated central vein $(\mathrm{CV})$ and the presence of Kupffer cells $(\mathrm{K})$ and narrow sinusoids $(\mathrm{S})$. H\&E x 400
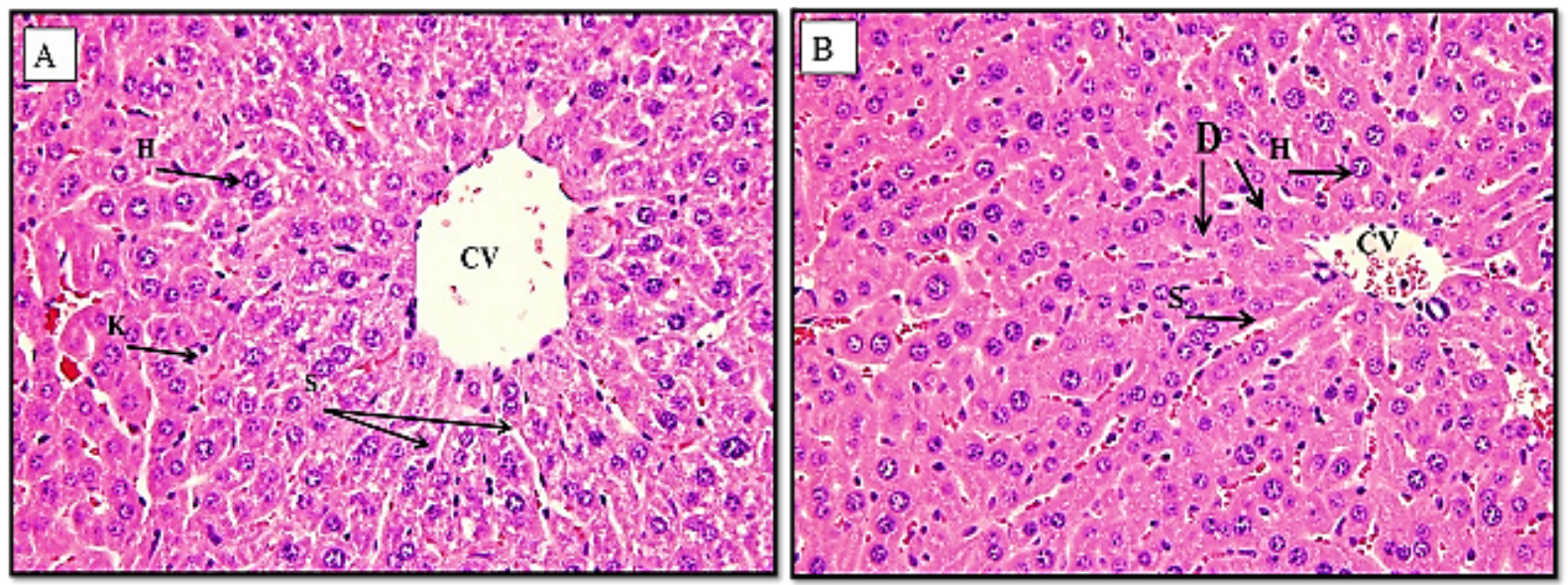

Figure 6. Photomicrograph of hepatic tissue of A) 5'FU-ME group, showing hepatocytes (H) rounded in shape, central vein (CV), activation of Kupffer cells (K) near to dilated sinusoidal spaces (S), and B) 5'FU-Sol group, showing damaged hepatocytes (D) that lack nuclei, hepatocytes (H), dilated central vein (CV) filled with blood and the presence of Kupffer cells (K) and dilated sinusoids (S). H\&E x 400 

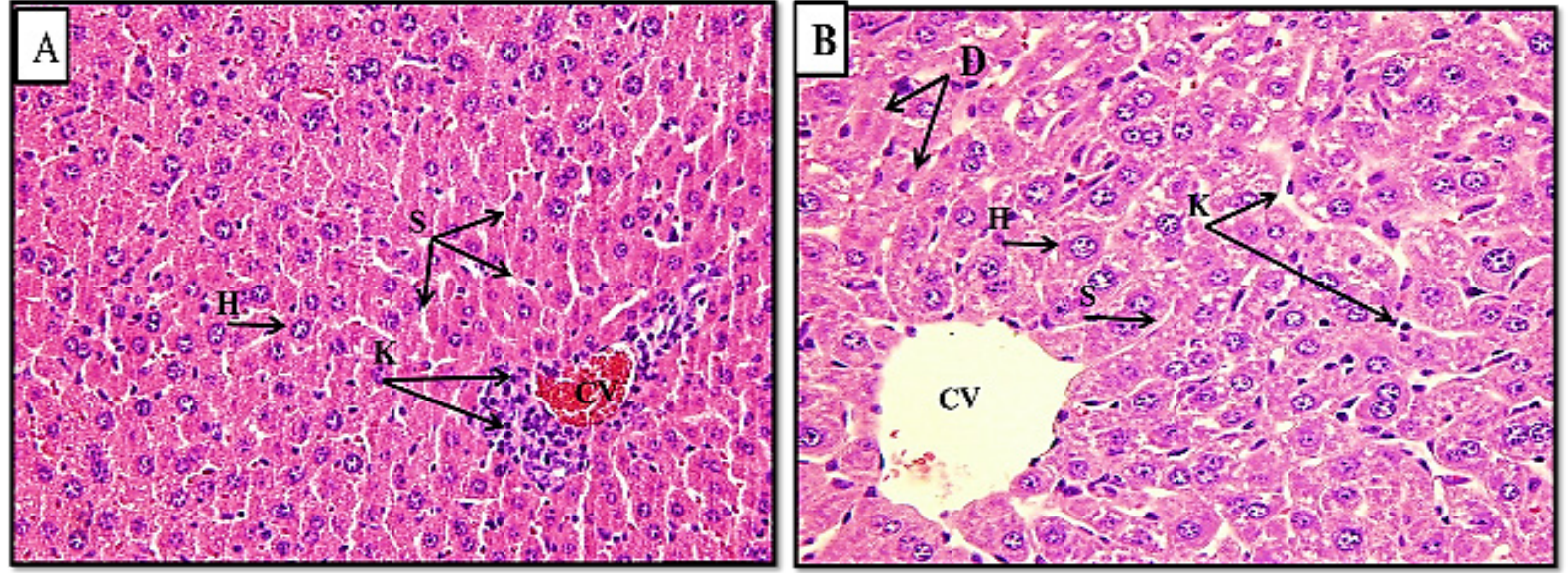

Figure 7. Photomicrograph of hepatic tissue of A) GEM+5'FU-ME group, showing hepatocytes $(\mathrm{H})$, central vein (CV) filled with blood, and the presence of Kupffer cells (K) around central vein, and narrow sinusoids (S), and B) GEM+5'FU-Sol group, showing damaged hepatocytes (D) that lost their regular shapes and their nuclei, hepatocytes $(\mathrm{H})$ rounded in shape, dilated central vein $(\mathrm{CV})$, activation of Kupffer cells (K) near to normal structure sinusoidal spaces (S). H\&E x 400

\section{Discussion}

Cancer is still the most fatal disease in the world [10]. In spite of the numerous anticancer drugs involved in cancer therapy, their serious adverse side effects, especially on the liver, need to be eliminated by proposing more delivery systems [11]. The overall effect of encapsulating both of the drugs, GEM and 5-FU, in ME have reduced their side effect on the liver enzymes and the liver tissues, although the drugs-loaded ME were administered orally while the solution formulations were applied intravenously into the mice. It seems that the ME has provided protection for the drugs from enzymatic degradation and improved their bioavailability [12]. In fact, it has been elaborated by Veltkamp [13], that GEM gets metabolized in the liver and thereby might be toxic to the liver. Additionally, 5-FU is might cause severe gastrointestinal toxicity, hematologic disturbance, and severe bone marrow deficiency, which remain significant limitations to its clinical use [14]. Several attempts were implemented to incorporate 5-FU with various chemotherapeutic agents, however, none of these combinations appeared to enhance the drug's efficacy [15].

Furthermore, all of the drugs were loaded inside the nanodroplets of the ME as the droplets sizes of the ME have either slightly or considerably enlarged when the drugs were added to indicate that the drugs were protected from degradation by the liver cells. The nanodroplets all of the ME formulations were negatively charged and homogeneously distributed, suggesting their potential role as a passive targeted antitumor agent. It has been found that a small to medium pegylated nanoparitcles have reduced plasma protein adsorption on their surface, reduced hepatic filtration, long blood residence time and high rate of extravasation tissues $[16,17]$.

\section{Conclusions}

It has been found that loading 5-FU and GEM in a ME has reduced their hepatotoxicity effect in the EAC-bearing mice. The small droplet sizes and negative charges of the ME provided protection for the drugs from degradation while preventing the death of the liver cells. It is recommended to implement further in vivo researches in order to detect the effect of the ME on the efficacy of the drugs.

\section{Acknowledgements}

The authors wish to express a sincere thanks and appreciation to King Abdulaziz City for Science and Technology for its financial support to the research project designated by number (P-S-36-134).

\section{REFERENCES}

[1] A. Sharma, R. Houshyar, P. Bhosale, J.I. Choi, R. Gulati, and C. Lall. Chemotherapy induced liver abnormalities: An imaging perspective, Clinical And Molecular Hepatology, Vol. 20, No. 3, 317-26, 2014.

[2] E. Moysan, G. Bastiat, and J.P. Benoit. Gemcitabine versus modified gemcitabine: a review of several promising chemical modifications, Pharmaceutics, Vol. 10, No. 2, 403444, 2013.

[3] S. Goindi, P. Arora, N. Kumar and A. Puri. Development of novel ionic liquid-based microemulsion formulation for dermal delivery of 5-Fluorouracil, AAPS PharmSciTech, Vol. 15, No. 4, 810-821, 2014. 
[4] S. Cascinu, R. Silva, S. Barni, R. Labianca, L. Frontini, E. Piazza, G. Pancera, P. Giordani, L. Giuliodori, M. Pessi, V. Fusco, G. Luporini, R. Cellerino, and G. Catalano. A combination of gemcitabine and 5-fluorouracil in advanced pancreatic cancer, a report from the Italian Group for the Study of Digestive Tract Cancer (GISCAD), British Journal of Cancer, Vol. 80, No. 10, 1595-1598, 1999.

[5] A. Novarino. Phase II study of cisplatin, gemcitabine and 5 -fluorouracil in advanced pancreatic cancer, Annals of Oncology, Vol. 15, No. 3, 474-477, 2004.

[6] R. Patel, M. Patel, K. Bhatt and B. Patel. Formulation consideration and characterization of microemulsion drug delivery system for transnasal administration of carbamazepine, Bulletin of Faculty of Pharmacy, Cairo University, Vol. 51, No. 2, 243-253, 2013.

[7] National Research Council (US) Committee for the Update of the Guide for the Care and Use of Laboratory Animals. Guide for the Care and Use of Laboratory Animals, 8th edition, National Academies Press (US), Washington (DC), 2011 .

[8] Y.H. Tsai, Y.H. Hsieh, Y.B. Huang, J.S. Chang, C.T. Huang, and P.C. Wu. Microemulsions for intravesical delivery of gemcitabine, Chemical \& Pharmaceutical Bulletin, Vol. 58, No. 11, 1461-1465, 2010.

[9] M. H. Alkhatib, H. M. Alkreathy, K. S. Balamash, and F. Abdu. Antitumor activity of doxorubicine-loaded nanoemulsion against ehrlich ascites carcinoma-bearing mice, Tropical Journal of Pharmaceutical Research, Vol. 15, No. 5, 937-943, 2016.

[10] D. Tila, S. Ghasemi, S. N. Yazdani-Arazi, and S. Ghanbarzadeh. Functional liposomes in the cancer-targeted drug delivery, Journal of Biomaterials Applications, vol. 30 no. 1, pp. 3-16, 2015.

[11] B. Liu, L. Ezeogu, L. Zellmer, B. Yu, N. Xu, and D. Joshua Liao, Protecting the normal in order to better kill the cancer, Cancer Medicine, Vol. 4, No. 9, 1394-403, 2015.

[12] V. B. Bhamere and B. A. Joshi. Microemulsions: novel drug delivery systems, International Journal of Universal Pharmacy and Bio Sciences, Vol. 3, No. 2, 2014.

[13] S. A. Veltkamp, D. Pluim, O. Van Tellingen, J. H. Beijnen, and J. H. M. Schellens. Extensive metabolism and hepatic accumulation of gemcitabine after multiple oral and intravenous administration in mice, Drug Metabolism and Disposition, Vol. 36, No. 8, 1606-1615, 2008.

[14] M. Cheng, B. He, T. Wan, W. Zhu, J. Han, B. Zha, H. Chen, F. Yang, Q. Li, W. Wang, H. Xu, and T. Ye. 5-Fluorouracil nanoparticles inhibit hepatocellular carcinoma via activation of the p53 pathway in the orthotopic transplant mouse model, PLOS One, Vol. 7, No. 10, 1-12, 2012.

[15] A. Teague, K.H. Lim, and A. Wang-Gillam. Advanced pancreatic adenocarcinoma: a review of current treatment strategies and developing therapies, Therapeutic Advances in Medical Oncology, Vol. 7, No. 2, 68-84, 2015.

[16] M. A. Gatoo, S. Naseem, M. Y. Arfat, A. M. Dar, K. Qasim, and S. Zubaird. Physicochemical properties of nanomaterials: implication in associated toxic manifestations, BioMed Research International, Vol. 2014, $8,2014$.

[17] Nuri Oh and Ji-Ho Park. Endocytosis and exocytosis of nanoparticles in mammalian cells, International Journal of Nanomedicine, Vol. 9, 51-63, 2014. 